\title{
Inefficient procurement processes undermine access to medicines in the Western Cape Province of South Africa
}

\author{
B P Magadzire, ${ }^{1}$ BSc, MPhil; K Ward,${ }^{2}$ BPharm, MPharm, PhD; H M J Leng, ${ }^{1}$ MPharm, PhD, MBA; D Sanders, ${ }^{1}$ MB ChB, MRCP, DCH, \\ DTPH, DSc (Hon Causa) \\ ${ }^{1}$ School of Public Health, Faculty of Community and Health Sciences, University of the Western Cape, Cape Town, South Africa \\ ${ }^{2}$ School of Pharmacy, Faculty of Natural Sciences, University of the Western Cape, Cape Town, South Africa
}

Corresponding author: B P Magadzire (bmagadzire@gmail.com)

Background. South Africa (SA) has experienced several stock-outs of life-saving medicines for the treatment of major chronic infectious and non-communicable diseases in the public sector.

Objective. To identify the causes of stock-outs and to illustrate how they undermine access to medicines (ATM) in the Western Cape Province, SA.

Methods. This qualitative study was conducted with a sample of over 70 key informants (frontline health workers, sub-structure and provincial health service managers). We employed the critical incident technique to identify significant occurrences in our context, the consequences of which impacted on access to medicines during a defined period. Stock-outs were identified as one such incident, and we explored when, where and why they occurred, in order to inform policy and practice.

Results. Medicines procurement is a centralised function in SA. Health service managers unanimously agreed that stock-outs resulted from the following inefficiencies at the central level: $(i)$ delays in awarding of pharmaceutical tenders; (ii) absence of contracts for certain medicines appearing on provincial code lists; and (iii) suppliers' inability to satisfy contractual agreements. The recurrence of stock-outs had implications at multiple levels: $(i)$ health facility operations; (ii) the Chronic Dispensing Unit (CDU), which prepacks medicines for over 300000 public sector patients; and (iii) community-based medicines distribution systems, which deliver the CDU's prepacked medicines to non-health facilities nearer to patient homes. For instance, stock-outs resulted in omission of certain medicines from CDU parcels that were delivered to health facilities. This increased workload and caused frustration for frontline health workers who were expected to dispense omitted medicines manually. According to frontline health workers, this translated into longer waiting times for patients and associated dissatisfaction. In some instances, patients were asked to return for undispensed medication at a later date, which could potentially affect adherence to treatment and therapeutic outcomes. Stock-outs therefore undermined the intended benefits of ATM strategies.

Conclusion. Addressing the procurement challenges, most notably timeous tender awards and supplier performance management, is critical for successful implementation of ATM strategies.

S Afr Med J 2017;107(7):581-584. DOI:10.7196/SAMJ.2017.v107i7.11356

Medicines shortages (stock-outs) have been cited as a complex global problem. Evidence suggests that the causes vary greatly and include issues that government agencies often do not have control over, such as supplier shortages. ${ }^{[1]}$ Stock-outs appear to be worsening with time globally, which presents a threat to patient wellbeing and could result in loss of life. ${ }^{[2]}$ At a global level, multi-stakeholder activities to evaluate the causes of stock-outs and to develop appropriate mitigating strategies have been initiated. ${ }^{[2]}$

Like many other countries, South Africa (SA) has experienced several stock-outs of life-saving medicines. There has also been a spate of media reports on stock-outs of essential medicines for treatment of HIV and major chronic non-communicable diseases. ${ }^{[3,4]}$ A 2012 publication by the National Department of Health $(\mathrm{NDoH})^{[5]}$ reported a $54 \%$ failure in compliance with measures addressing availability of medicines and recommended priority attention to supply chain management. Local research has found that confidence in the public sector has waned as a result of stock-outs. ${ }^{[6]}$

These and other ongoing challenges in the SA medicines supply chain served as the impetus to investigate the supply chain and to identify barriers to access to medicines (ATM) and existing strategies for improving ATM under the Accessing Medicines in Africa and South Asia project. ${ }^{[7]}$ In this quest, we acknowledged that ATM barriers are multi-layered, with overlapping and interconnected causes, ${ }^{[2,8]}$ and that the supply chain in SA is diverse and complex. Notwithstanding these complexities, it is expected to reliably supply pharmaceuticals from manufacturers to provincial depots and subdepots (in some cases), and directly or indirectly to all levels of health facilities, centralised dispensaries and other non-health facilities. This is crucial for meeting the growing demand for medicines, particularly for treatment of chronic diseases, among others. ${ }^{[9]}$ Given the various interconnections in the supply chain, our approach was to investigate how the complex interplay of ATM dimensions, including pharmaceutical regulation, medicines distribution and demandside barriers, influenced ATM in SA. We selected tracer medicines commonly used in the public sector that could aid in identifying the relevant issues in the supply chain. These were rifampicin (antituberculosis), metformin (antidiabetic, type 2), lamivudine (antiretroviral), fluoxetine (antidepressant) and oxytocin (labour induction). ${ }^{[7]}$

Previous articles emanating from this research reported on policy implications for medicine registration ${ }^{[10]}$ and equity in the geographical distribution of community pharmacies in SA. ${ }^{[11]}$ We also identified supply and demand barriers to $\mathrm{ATM}^{[12]}$ and existing strategies for mitigating these barriers. ${ }^{[12,13]}$ In the Western Cape 
(WC) Province, we identified two distinct strategies for improving ATM: the Chronic Dispensing Unit (CDU) and community-based medicines delivery models. The CDU, a model for centralised dispensing of medicines in the public sector, was introduced to address the shortage of pharmacists, reduce patient waiting times and decongest health facilities. ${ }^{[13-15]}$ Community-based medicines delivery models were aimed at distributing medicines closer to patients' homes, thereby addressing barriers associated with physical accessibility and affordability of transport costs. These models are fully described elsewhere. ${ }^{[16-18]}$ Despite these advances in the WC, public sector stock-outs hampered the efficiency of ATM strategies and disrupted service provision. Among the reasons offered for stock-outs were inefficiencies in procurement, a centralised function. In this article we therefore demonstrate how inefficient procurement processes have undermined ATM.

\section{Medicines procurement in SA}

Procurement of medicines in SA is centralised and managed by the $\mathrm{NDoH}$, with some variation in organisation between procurement of HIV medicines and that of other medicines. Pharmaceutical tenders are advertised, awarded and monitored by the NDoH. Provinces submit quantification figures to the $\mathrm{NDoH}$ for the contract period, where the figures are then aggregated. Contracted suppliers deliver directly to depots and in some cases (for selected items) directly to the CDU and health facilities.

\section{Methods}

This qualitative study was conducted between 2012 and 2014 with a purposeful sample of over 70 key informants representing frontline health workers, sub-structure and provincial level health service managers.

\section{Application of the critical incident technique}

We employed the critical incident technique ${ }^{[19]}$ to identify occurrences that have special significance in our context. The method focuses on gathering data that are potentially useful in solving practical problems. Critical incidents were described by Flanagan ${ }^{[19]}$ as:

- an extreme behaviour, either outstandingly effective or ineffective with respect to attaining the general aims of an activity

- an incident that is complete in itself to permit inferences and predictions to be made, and the consequences of which are sufficiently definite to leave little doubt concerning its effects.

In this research, the critical incident was defined as any such incident having special significance in our context.

Data collection. The critical incident technique consists of a set of procedures for collecting information that allow participants to 'tell the story' (what) and then generate details by posing probing 'when', 'where' and 'why' questions. The technique is commonly used to collect data on observations previously made that are reported from memory - which is usually satisfactory when the incidents reported are fairly recent. However, in some situations adequate coverage cannot be obtained if only very recent incidents are included. ${ }^{[19]}$ As such, we requested the informants to reflect on a longer period ( 5 years) to identify incidents positively or negatively affecting ATM. To allow for this detailed exploration, we used a semi-structured interview guide containing open-ended questions as a data collection tool.

Data analysis. Our process involved listing the incidents identified, then making a judgement based on the principles of the critical incident technique as described earlier. We then developed descriptions of the selected incidents.

\section{Ethics approval and consent to participate}

Ethics approval for this study was granted by the Senate Research Committee of the University of the Western Cape (ref. no. 11/7/8). Consent to interview and record interviews was obtained from participants. They were also informed of their right to withdraw from the interview at any time.

\section{Results}

\section{Overview of the general state of medicines}

\section{availability in the WC}

Informants recognised the positive role by the WC Department of Health in instituting measures to minimise stock-outs in the province. They reported that in general, stock-outs at the provincial depot had reduced significantly from over 160 out-of-stock items each week a few years before to an average of $10-20$ items at the time of the study, some of which could be substituted by the use of different pack sizes of the same medicine. Frontline health workers received regular updates on the stock situation from the depots, which enabled them to make alternative arrangements where possible. Sometimes buffer stock was available in the health facilities, enabling pharmacists to continue dispensing activities without disruption in the event of stock-outs at the depot.

However, poor forecasting at the level of health facilities was sometimes regarded as a challenge, particularly during times of policy changes or strategic service delivery decisions that led to increased demand for specific medicines and medical devices without the necessary adjustments to the quantification and ordering processes. Such events occurred when health managers developed programmes or campaigns and neglected to liaise timeously with the provincial supply chain team, which could in turn result in stock-outs. One such incident was described by a pharmacy manager as follows:

'... last month we had a problem with Accu-check sticks [for blood glucose testing] because the province started testing everyone for glucose and nobody told the depot "Listen, we need more ...", and the depot didn't know to tell the company [supplier], so now we've got this snowball effect and ... we don't have enough to give the patient.'

Although the above example referred to medical devices, similar experiences occurred with tracer medicines, and the informant raised it in that context.

Besides province-specific issues, processes at central level caused stock-outs at particular time periods. Two of our tracer medicines, i.e. metformin and rifampicin, were affected by these processes, and so were other medicines. This in turn had implications for ATM.

\section{Perceived causes of procurement inefficiencies}

Stock-outs were attributed to: (i) delay in award of pharmaceutical tenders; (ii) removing national contracts for certain medicines on provincial code lists; and (iii) supplier failure to meet contract obligations. We elaborate on these three factors below, and discuss the implications of stock-outs for ATM strategies in the WC.

\section{Delay in award of pharmaceutical tenders}

Delayed award of pharmaceutical tenders by the NDoH emerged as one of the key challenges facing the pharmaceutical system. Some informants attributed tender challenges to a lack of manpower in the $\mathrm{NDoH}$ to timeously renew tenders - a function previously performed by National Treasury. During our research, we witnessed the knockon effect of late tender awards in 2012 and 2014. Informants reported that this was a common occurrence every 2 years when 
pharmaceutical contracts expired. By 2014, some informants had witnessed the impact of late tender awards over a number of cycles, which led to an increase in the number of out-of-stock items.

As indicated by one informant:

'... we had dues out [out-of-stock medicines] down to about 10 to 15 at one stage for quite a long period at depot side. Now it's started creeping up to $60 \ldots$ in a crisis 2 years ago it was over 100. But it is on the increase because of these tender problems.' (Senior provincial manager)

Another informant added:

'Every 2 years when the contracts expire we go through these (stock) problems ... at the moment we're sitting with a 66 item dues-out at depot [medicines unavailable from the depot], and this is because of the tendering system ... And that's the biggest gap in the system ... they [the $\mathrm{NDoH}$ ] know exactly when the tender is going to end, but the process takes so long. Then they have to ask current tender companies to extend their contract for 1 or 2 months. The company's response could be, "I don't want to do it, and I don't have the capacity." Then they have to look for another company and this usually leads to problems.' (Mid-level manager, district substructure)

In an effort to understand reasons for suppliers' unwillingness to extend contracts, one informant shared the following insights:

'I think there is a lack of understanding with our tender guys [procurement officials] that these pharmaceutical manufacturers sometimes plan 2 years in advance. And when they plan, when they have been allocated the contract that obviously has a major impact on their production planning - and we must remember that they don't produce only one item or two items, they've got a variety of products that they manufacture. They have timeslots and certain machines for all these, and one machine might be used for two different products. So there are major challenges for them as well, but from a planning point of view it really has a negative effect on these guys [suppliers].' (Mid-level manager, medicines procurement)

Informants also added that suppliers tend to downscale production in the last few months of their contract because of uncertainties around successful rebidding. In view of the tough economic climate, it is no longer financially viable for suppliers to produce excess stock, whereas in the past they held significant amounts of buffer stock, allowing them the flexibility to extend their contracts. Suppliers are also sometimes reluctant to extend their contracts because of the unplanned production upscaling and the associated risk of being penalised should they fail to meet the prescribed timelines. Interim options to source large volumes of medicine from alternative pharmaceutical companies that are not contracted to the $\mathrm{NDoH}$ are limited, since these companies tend to downscale manufacturing of a particular medicine if they fail to secure a national contract.

\footnotetext{
Absence of national contracts for medicines on the provincial code list

According to informants, at times there was poor communication between provincial and national stakeholders. A case in point: about 70 items were excluded from the most recent national tender at the time of the study (2014) without first consulting provinces, which was the usual practice in order to allow health providers to prepare for the change. Informants thought this action was a result of efforts by the NDoH to align medicines tenders to the Essential
}

Medicines List. At the same time, a decision was taken to include only one item per medicine class, which placed a strain on the industry to increase production of these items and also meant that there were no alternatives for the provinces in the event of shortages. While provinces could lobby for the medicines to be placed back on national tender and/or to procure directly from suppliers (sometimes involving importation of medicines), these processes often took a long time, with resultant delays in supply. In other cases, guideline modification through the Provincial Pharmacy and Therapeutics Committees was required to allow for medicines substitution. The positive note reported by our informants was that the $\mathrm{NDoH}$ acknowledged the risk of only including one item per class.

\section{Failure by suppliers to meet demand}

Suppliers failed to meet contractual obligations due to: (i) global shortages of active pharmaceutical ingredients (APIs) (e.g. the shortage of APIs for antituberculosis medicines during the study period); and (ii) lack of capacity, especially during the start-up phase, after a contract is awarded, when demanders procure large volumes to compensate for stock-outs imposed by delayed contracting. General capacity constraints also became evident during the course of the contract when a supplier failed to meet demands, and although these incidents were reported to the $\mathrm{NDoH}$, it was not unusual for the contractor to be reappointed during the next tender cycle. One informant said:

'We are tied [left with no option] ... the most we can do is inform the National Department of Health that they have been bad suppliers ... but history has shown us and our experience has shown us that those suppliers do get reappointed with contracts again and we sit with the same problems again.' (Midlevel manager, medicines procurement)

When a supplier provides a substandard/inferior product and a medicine recall ensues, a severe disruption in supply may result. An incident cited by informants was when the state received supplies of rifampicin tablets containing substantially less than the specified amount of active ingredient.

\section{Implications of stock-outs for ATM strategies}

Strategies for improving ATM in the WC were affected by stock-outs. First, the CDU system, which dispenses medicines for over 300000 patients, operates on a set production schedule and stock received after commencement of the production process cannot be introduced into the dispensing system. Stock-outs resulted in batches of patient medicine parcels (PMPs) being dispatched to health facilities without all the prescribed medicines. Consequently, pharmacists at health facilities manually dispensed the missing medicines from buffer stock or provided alternative therapy under the authorisation of a prescriber. Owing to the high burden of chronic diseases in the province, this meant that several hundreds of PMPs at each health facility required additional medicines. While it was commendable that health facilities sometimes had buffer stock that allowed them to minimise the impact of stock-outs on patients, workload in the pharmacies increased due to the additional dispensing tasks and caused frustration among pharmacists. Accordingly, patient waiting times increased. In instances where no buffer stock was available, patients had to return at a later date when the medicine was expected to be available. Also, community-based services rely on the CDUs' prepacked PMPs, and these services were affected when stock-outs occurred. Stock-outs caused service interruption, forcing patients who benefited from community-based services to attend the health 
facility for their medicine supply. This imposed additional transport costs on patients and is likely to have impacted negatively on their welfare.

Stock-holding at depots (up to 6 months) and health facilities (4 - 8 weeks) generally created a buffer to procurement and other challenges in medicine supply, ensuring that patients had access to their medicines. In recognising the importance of maintaining buffer stock, there were some concerns that the recommendation from the $\mathrm{NDoH}$ for the public sector to migrate to a 'push' model, which supplies pre-defined and standard volumes of medicine down to health facilities based on demand planning, as opposed to a 'pull' model, which relies on orders from health facilities, could preclude holding buffer stock.

\section{Discussion}

The challenges imposed by stock-outs are becoming more evident against the backdrop of increased demand for chronic disease treatment in SA, ${ }^{[20]}$ but they are not new. ${ }^{[21]}$ While some studies have reported that pharmaceutical systems in low- and middle-income countries are generally strained and weak, ${ }^{[22-24]}$ there has been a limited focus on procurement processes, yet the critical incidents identified in our study were linked to this issue.

The interconnected nature of different levels of the health system (provincial and national) was clear. Based on the issues raised by this research, minimising stock-outs requires action at a national level, where procurement takes place, to ensure that tenders are awarded timeously and supplier performance is monitored. Also, the provinces (not only the WC) have a responsibility to provide accurate forecasts to the NDoH.

Existing ATM strategies ${ }^{[13-17]}$ depend on a consistent, secure and reliable source of medicines to function optimally, yet they are regularly interrupted by stock-outs. Addressing the prevailing challenges will be of benefit to the country as a whole given that medicines will continue to play an integral role in the health system, not only of the individual provinces but also to achieve the ATM goals under the proposed National Health Insurance scheme in SA. ${ }^{[25]}$

\section{Conclusion}

Addressing causes of stock-outs, starting at the national level, is critical for successful implementation of ATM strategies in SA and to ensure that patient care is not adversely affected. The recurrence of stock-outs due to predictable events warrants continued attention to the underlying issues. Addressing the procurement challenges, most notably timeous tender awards and supplier performance management, is critical for successful implementation of ATM strategies.

Acknowledgements. We thank the Western Cape Department of Health for allowing us to learn from their experience.

Author contributions. KW, HMJL and DS contributed to the conceptualisation of the research. KW, HMJL and BPM conducted the field research. All authors contributed to the conceptualisation of the manuscript. BPM undertook qualitative data analysis and drafted the manuscript. All authors contributed to the intellectual content of the article. BPM finalised the article. All authors read and approved the final manuscript.
Funding. This research and the involvement of co-authors was made possible by funding from the European Union Seventh Framework Programme Theme: Health-2009-4.3.2-2 (grant no. 242262) under the title 'Accessing Medicines in Africa and South Asia', which was concluded in 2013. Subsequent work was made possible by the first author's doctoral funding from the South African Research Chair Initiative in Health Systems, Complexity and Social Change at the University of the Western Cape, the Belgian Development Cooperation and an African Doctoral Dissertation Research Fellowship award offered by the Africa Population and Health Research Centre in partnership with the International Development Research Centre.

Conflicts of interest. None.

1. Gray A, Manasse HR jun. Shortages of medicines: A complex global challenge. Bull World Health Organ 2012;90(3):158-158A. http://dx.doi.org/10.2471/BLT.11.101303

2. International Pharmaceutical Federation (FIP). Report of the International Summit on Medicines Shortage. Toronto: FIP, 2013.

3. Gray A. Medicines shortages - unpicking the evidence from a year in South Africa. Australas Med J 2014;7(5):208-212. http://dx.doi.org/10.4066/AMJ.2014.1932

Bateman C. Drug stock-outs: Inept supply-chain management and corruption. S Afr Med J 2013;103(9):600-602. http://dx.doi.org/10.7196/SAMJ.7332

5. Health Systems Trust. The National Health Care Facilities Baseline Audit: National Summary Report. Durban: HST, 2012.

6. Goudge J, Gilson L, Russell S, Gumede T, Mills A. Affordability, availability and acceptability barriers to health care for the chronically ill: Longitudinal case studies from South Africa. BMC Health Serv Res 2009;9(1):75. http://dx.doi.org/10.1186/1472-6963-9-75

7. Accessing Medicines in Africa and South Asia project. http://www.amasa-project.eu/ (accessed 12 May 2016).

8. Bigdeli M, Jacobs B, Tomson G, et al. Access to medicines from a health system perspective. Health Policy Plan 2013;28(7):692-704. http://dx.doi.org/10.1093/heapol/czs108

9. Deuboué Tchialeu RI, Yaya S, Labonté R. Health systems readiness to manage the hypertension epidemic in primary health care facilities in the Western Cape, South Africa: A study protocol. JMIR Ridemic in primary health care facilities in the Western Cape,
Res Protoc 2016;5(1):e35. http://dx.doi.org/10.2196/resprot.5381

10. Leng HMJ, Sanders D, Pollock AM. Pro-generics policies and the backlog in medicines registration in South Africa: Implications for access to essential and affordable medicines. GaBI J 2015;4(2):58-63.
in in South Africa: Implications for access to esse
http://dx.doi.org/10.5639/gabij.2015.0402.014

11. Ward K, Sanders D, Leng H, Pollock AM. Assessing equity in the geographical distribution of community pharmacies in South Africa in preparation for a national health insurance scheme. Bull World Health Organ 2014;92(7):482-489. http://dx.doi.org/10.2471/BLT.13.130005

12. Magadzire BP, Budden A, Ward K, Jeffery R, Sanders D. Frontline health workers as brokers: Provider perceptions, experiences and mitigating strategies to improve access to essential medicines in South Africa. BMC Health Serv Res 2014;14(1):520. http://dx.doi.org/10.1186/s12913-014-0520-6

13. Magadzire BP, Marchal B, Ward K. Improving access to medicines through centralised dispensing in the public sector: A case study of the Chronic Dispensing Unit in the Western Cape Province, South Africa. BMC Health Serv Res 2015;15(1):513. http://dx.doi.org/10.1186/s12913-015-1164-x

14. Du Plessis J. The Chronic Dispensing Unit. S Afr Pharm J 2008;75(9):46-47.

15. Du Toit J, Dames S, Boshoff R. Centralised dispensing - an affordable solution. S Afr Pharm J 2008; 75(10):18-20.

16. Grimsrud A, Sharp J, Kalombo C, Bekker LG, Myer L. Implementation of community-based adherence clubs for stable antiretroviral therapy patients in Cape Town, South Africa. J Int AIDS Soc 2015;18(19984):19984. http://dx.doi.org/10.7448/IAS.18.1.19984

17. Wilkinson LS. ART adherence clubs: A long-term retention strategy for clinically stable patients receiving antiretroviral therapy. South Afr J HIV Med 2013;14(2):48-50. http://dx.doi.org/10.7196/ SAJHIVMED. 924

18. Luque-Fernandez MA, van Cutsem G, Goemaere E, et al. Effectiveness of patient adherence groups as a model of care for stable patients on antiretroviral therapy in Khayelitsha, Cape Town, South Africa. PLoS One 2013;8(2):e56088. http://dx.doi.org/10.1371/journal.pone.0056088.g001

19. Flanagan J. The critical incident technique. Psychol Bull 1954;51(4):327-358. http://dx.doi.org/10.1037/ h0061470

20. Whiteside A, Cohen J, Strauss M. Reconciling the science and policy divide: The reality of scaling up antiretroviral therapy in South Africa. South Afr J HIV Med 2015;16(1), Art. \#355, 5 pages. http:// dx.doi.org/10.4102/sajhivmed.v16i1.355

21. Steyn F, Schneider H, Engelbrecht MC, van Rensburg-Bonthuyzen EJ, Jacobs N, van Rensburg DH. Scaling up access to antiretroviral drugs in a middle-income country: Public sector drug delivery in 1-6. http://dx.doi.org/10.1080/09540120903131138

22. Tetteh E. Creating reliable pharmaceutical distribution networks and supply chains in African countries: Implications for access to medicines. Res Social Adm Pharm 2009;5(3):286-297. http:// dx.doi.org/10.1016/j.sapharm.2008.08.001

23. Berhanemeskel E, Beedemariam G, Fenta TG. HIV/AIDS related commodities supply chain management in public health facilities of Addis Ababa, Ethiopia: A cross-sectional survey. J Pharm Policy Pract 2016;9:11. http://dx.doi.org/10.1186/s40545-016-0060-z

24. Quick JD, Boohene NA, Rankin J, Mbwasi RJ. Medicines supply in Africa. BMJ 2005;331(7519):709710. http://dx.doi.org/10.1136/bmj.331.7519.709

25. National Department of Health. National Health Insurance for South Africa: Towards Universal Health Coverage. Pretoria: NDoH, 2015

Accepted 28 March 2017. 